Check for updates

Cite this: RSC Adv., 2017, 7, 54369

Received 22nd September 2017 Accepted 18th November 2017

DOI: $10.1039 / c 7 r a 10506 a$

rsc.li/rsc-advances

\section{p-JAK2 plays a key role in catalpol-induced protection against rat intestinal ischemia/ reperfusion injury $\dagger$}

\author{
Mengqiao Lian, $\stackrel{t}{t}^{\mathrm{a}}$ Yuchao Sun, $\stackrel{+}{\mathrm{t}}^{\mathrm{a}}$ Yuan Lin, (D) *ac Jin Wen, ${ }^{\mathrm{a}}$ Marwan Almoiliqy, ${ }^{\mathrm{a}} \mathrm{Bin} \mathrm{Xu},{ }^{a}$ \\ Yanli Li, ${ }^{a}$ Ming $\mathrm{Xu}{ }^{\text {*a }}$ Dapeng Chen, (D) ${ }^{\mathrm{b}}$ Zeyao Tang ${ }^{\mathrm{a}}$ and Li Wang ${ }^{\mathrm{a}}$
}

Catalpol, an iridoid glucoside isolated from the radix of Rehmannia glutinosa Libosch, is found to have a wide variety of biological activities. Based on our pre-experiments, we proposed that catalpol pretreatment could protect against intestinal ischemia/reperfusion (I/R) injury and the JAK2/STAT3 signaling pathway might play an important role in the protection. Both in vivo intestinal I/R-injured rats and in vitro hypoxia/reoxygenation (H/R)-injured IEC-6 cells were used in the present study. The results confirmed our proposal. The in vivo results showed that catalpol $\left(25,50 \mathrm{mg} \mathrm{kg}^{-1}\right)$ significantly attenuated rat intestinal I/R injury by decreasing pro-inflammatory cytokines, reducing oxidative stress, and restoring intestinal barrier function without affecting the corresponding controls. Catalpol pretreatment significantly increased the $\mathrm{Bcl}-2 / \mathrm{Bax}$ ratio, and decreased cleaved caspase3, p-JAK2, and p-STAT3 protein expression without affecting total JAK2 and STAT3 in vivo and in vitro, showing that catalpol inhibited apoptosis through selectively inhibiting $p$ JAK2 and p-STAT3. In vitro studies indicated that catalpol $(40 \mu \mathrm{M})$ pretreatment significantly and selectively inhibited p-STAT3 nuclear translocation and transfection of JAK2 siRNA significantly decreased JAK2, p-JAK2, STAT3, p-STAT3, and other apoptosis-related proteins in H/R-injured IEC-6 cells, suggesting that JAK2 siRNA partially simulated the effects of catalpol on apoptosis-related proteins. JAK2 siRNA + catalpol could not further decrease JAK2, p-JAK2, STAT3, p-STAT3, and other apoptosis-related proteins, suggesting that inhibition of the JAK2/STAT3 signaling pathway plays a key role in catalpolinduced protective effects. Our results suggest that p-JAK2 is the key target in catalpol-induced protection against intestinal I/R injury, providing information for further translational studies.

\section{Introduction}

Intestinal ischemia/reperfusion (I/R) injury is a common clinical setting, particularly in acute mesenteric ischemia, septic shock, vascular surgery, and trauma. ${ }^{\mathbf{1} 2}$ Intestinal I/R may cause inflammation, oxidative stress, and apoptosis, induce intestinal barrier dysfunction, and facilitate bacteria and endotoxin transport into the blood, leading to intestinal infection and even multiple organ dysfunction syndrome..$^{3-5}$ Multiple signaling pathways are found to be correlated to $\mathrm{I} / \mathrm{R}$ injury, including Nrf2/HO-1, ${ }^{6,7}$ SIRT1, ${ }^{8,9}$ PI3K/AKT-eNOS, ${ }^{10,11}$ JAK2/ STAT3, ${ }^{\mathbf{1 2}}$ and P38 MAPK $^{\mathbf{1 3}, \mathbf{1 4}}$ signaling pathways.

To effectively ameliorate I/R-induced tissue injuries and reveal the correlated mechanisms, natural health promoting

\footnotetext{
${ }^{a}$ Pharmaceutical College, Dalian Medical University, Dalian, 116044, China. E-mail: dmuppliny@dlmedu.edu.cn; linyuandmu2008@qq.com; mingx2003@hotmail.com ${ }^{b}$ Laboratory Animal Center, Dalian Medical University, Dalian, 116044, China 'Zhongshan College, Dalian Medical University, Dalian, 116085, China $\dagger$ Electronic supplementary information (ESI) available. See DOI: 10.1039/c7ra10506a

\$ These authors contributed equally to this work.
}

compounds which possess diverse biological effects are believed to be potentially beneficial. Rehmannia glutinosa Libosch (known as Dihuang in China) has been used for centuries as an oriental traditional dietary herb or functional food. ${ }^{15,16}$ Catalpol, an iridoid glucoside of the main active component in the radix from Rehmannia glutinosa Libosch, ${ }^{17,18}$ is found to have a wide variety of biological activities including anti-oxidative, anti-inflammatory, anti-cancer, hypoglycemic, and analgesic effects. ${ }^{19,20}$ Catalpol is found to have protective effects against tissue injuries including liver, kidney, and brain injuries. ${ }^{19,21,22}$ Catalpol also shows a strong anti-microbial effect against intestinal infection caused by pathogens during intestinal I/R. ${ }^{19,23}$ However, whether catalpol can effectively protect against intestinal I/R injury remains unknown.

Based on our pre-experiments, we proposed that catalpol pretreatment could protect against intestinal $\mathrm{I} / \mathrm{R}$ injury by inhibiting I/R-induced inflammation, oxidative stress, apoptosis, and intestinal barrier dysfunction, and JAK2/STAT3 signaling pathway might play an important role in catalpolinduced protective effects. The present study was designed to verify our proposal by using rat $\mathrm{I} / \mathrm{R}$ injury model in vivo and 
using hypoxia/reoxygenation (H/R)-injured rat small intestinal crypt epithelial cells (IEC-6 cells) in vitro.

\section{Materials and methods}

\subsection{Chemical reagents}

Catalpol (purity quotient: $\geq 97 \%$ ) was purchased from Aladdin (Shanghai, China). Reagent kits for detection of malondialdehyde (MDA), superoxide dismutase (SOD), glutathione (GSH), and myeloperoxidase (MPO) were purchased from Nanjing Jiancheng Institute of Biotechnology (Nanjing, China). Doubleantibody sandwich ELISAs for detection of interleukin-6 (IL-6), interleukin-1 $\beta$ (IL-1 $\beta)$, tumor necrosis factor- $\alpha$ (TNF- $\alpha$ ), intestinal fatty acid binding protein (I-FABP), and diamine oxidase (DAO) were purchased from Shanghai Lengton Bioscience Co. LTD (Shanghai, China). Dulbecco's Minimum Essential Medium (DMEM) was bought from Gibco (CA, USA). Fetal bovine serum (FBS) was obtained from ScienCell research laboratories (CA, USA). The protein extraction kits and bicinchoninic acid (BCA) protein assay kits were obtained from Beyotime Institute of Biotechnology (Jiangsu, China). 4',6Diamidino-2-phenylindole (DAPI) was purchased from Sigma Chemical Co. (St.Louis, MO, USA). Cell counting kit-8 (CCK-8) was provided by Biotool (Shanghai, China).

\subsection{Animals}

Male Sprague-Dawley (SD) rats weighting 200-220 g were obtained from the Experimental Animal Center of Dalian Medical University (Certificate of Conformity: No. SCXK 2008-2002). All rats were fed for one week before experiment in a room temperature of $22 \pm 2{ }^{\circ} \mathrm{C}$ with $12 \mathrm{~h}$ light-to-dark cycle and provided standard food and water ad libitum. The experimental protocol was carried out based on the Declaration of Helsinki and supported by Dalian Medical University Animal Care and Ethics Committee. Human subjects are not involved in the present study.

\subsection{Establishment of intestinal $I / R$ injury rat model}

Before surgery, rats were fasted for $12 \mathrm{~h}$ with free access to water and then were anesthetized with sodium pentobarbital (50 $\mathrm{mg} \mathrm{kg}^{-1}$ body weight, i.p.) and subjected to a $2 \mathrm{~cm}$ midline abdominal incision. The rat intestinal $\mathrm{I} / \mathrm{R}$ model was established by superior mesenteric artery (SMA) occlusion as previously described. ${ }^{24}$ The SMA was isolated and clamped with an atraumatic microvascular clamp for $1 \mathrm{~h}$, and then, the clamps were removed to allow $2 \mathrm{~h}$ reperfusion. ${ }^{3}$ After reperfusion, blood samples were withdrawn via abdominal aorta and small intestinal segments were prepared for analysis.

Catalpol was dissolved in normal saline (NS), and rats were administered catalpol or NS intragastrically (i.g.) once daily in a volume of $2 \mathrm{~mL} \mathrm{~kg}^{-1}$. Rats were assigned randomly into five groups ( $n=8$ in each group): (1) sham group: rats were administered i.g. NS for three consecutive days and underwent isolation of SMA without occlusion; (2) catalpol $(\mathrm{H})+$ sham group: rats were pretreated with catalpol at a dose of $50 \mathrm{mg} \mathrm{kg}^{-1}$ once daily for three consecutive days before surgery and underwent isolation of SMA without occlusion; (3) I/R group: rats were administered i.g. NS and were subjected to $1 \mathrm{~h}$ intestinal ischemia and $2 \mathrm{~h}$ reperfusion; (4) catalpol (L) + I/R group: rats were pretreated with catalpol at a dose of $25 \mathrm{mg} \mathrm{kg}^{-1}$ once daily for three consecutive days before surgery and were subjected to $1 \mathrm{~h}$ intestinal ischemia and $2 \mathrm{~h}$ reperfusion; (5) catalpol $(\mathrm{H})+$ $\mathrm{I} / \mathrm{R}$ group: rats were pretreated with catalpol at a dose of $50 \mathrm{mg}$ $\mathrm{kg}^{-1}$ once daily for three consecutive days before surgery and were subjected to $1 \mathrm{~h}$ intestinal ischemia and $2 \mathrm{~h}$ reperfusion.

\subsection{Cell culture and $H / R$}

IEC-6 cells were cultured in Dulbecco's modified Eagle's medium (DMEM, Gibco) containing 10\% fetal bovine serum (FBS), 1\% penicillin/streptomycin, and $0.1 \mathrm{U} \mathrm{mL}^{-1}$ bovine insulin. IEC-6 cells were maintained in a humidified atmosphere containing $5 \% \mathrm{CO}_{2}$ at $37{ }^{\circ} \mathrm{C}$. To stimulate hypoxic conditions, IEC-6 cells were incubated in a microaerophilic system with $5 \% \mathrm{CO}_{2}$ and $1 \% \mathrm{O}_{2}$ and balanced with $94 \% \mathrm{~N}_{2}$ for $4 \mathrm{~h}$. The cells were cultured for $3 \mathrm{~h}$ under normoxic conditions to achieve reoxygenation.

\subsection{Cell viability assay}

IEC-6 cells were seeded in 96-well plates and grown overnight. The cells were treated with gradient dilution of catalpol $(2.5,5$, $10,20,40,80,160 \mu \mathrm{M})$ and incubated for $6 \mathrm{~h}, 12 \mathrm{~h}$, and $24 \mathrm{~h}$ at $37{ }^{\circ} \mathrm{C}$ with $5 \% \mathrm{CO}_{2}$ before $\mathrm{H} / \mathrm{R}$. According to the protocol, CCK-8 regent $(10 \mu \mathrm{L})$ was added to each well for $1 \mathrm{~h}$ incubation at $37^{\circ} \mathrm{C}$, which was measured at $450 \mathrm{~nm}$ using a microplate reader (PerkinElmer, USA).

\subsection{Histological analysis and in situ TUNEL staining}

After sacrifice, the isolated rat small intestinal segments were inflated in $4 \%$ paraformaldehyde solution. Paraffin sections (4 $\mu \mathrm{m}$ thickness) of intestine were prepared and stained with haematoxylin and eosin ( $\mathrm{H} \& \mathrm{E})$. The histopathological score was selected for evaluation of lesions in intestinal tissues by using Chiu's method. Grade 0 represents normal villi and grades 1-5 indicates increments of severity of injury (grade 1: capillary congestion; grade 2: moderate lifting of epithelial layer; grade 3: severe progressive lifting of epithelial layer; grade 4; denuded villi, dilated capillaries; grade 5: digestion \& disintegration of lamina propria, hemorrhage and ulceration). Determination of apoptosis in tissue was performed using an in situ cell death detection kit (Keygen Biotech, China) according to the manufacturer's instructions.

\subsection{In vitro apoptotic index determination}

Measurement of in vitro apoptotic cell death was performed using a TUNEL Apoptosis Detection Kit from Roche. IEC-6 cells were fixed in $4 \%$ paraformaldehyde (PFA) for $30 \mathrm{~min}$ at room temperature. Then, the fixed cells were incubated with $0.1 \%$ TritonX-100 for $10 \mathrm{~min}$. After washing cells with phosphatebuffered saline (PBS), $50 \mu \mathrm{L}$ of TUNEL reaction mixture was added, and cells were incubated at $37{ }^{\circ} \mathrm{C}$ for $1 \mathrm{~h}$ and then observed under a fluorescence microscope (Olympus, Japan). 
Acridine orange/ethidium bromide $(\mathrm{AO} / \mathrm{EB})$ staining indicated that the viable cells exhibited bright green and the apoptotic cells showed orange color. IEC-6 cells were stained with $\mathrm{AO}\left(200 \mu \mathrm{g} \mathrm{mL} \mathrm{mL}^{-1}\right)$ and $\mathrm{EB}\left(200 \mu \mathrm{g} \mathrm{mL}^{-1}\right)$ for $10 \mathrm{~min}$ and then washed with PBS to remove background staining. And the cells were then observed under a fluorescence microscope.

\subsection{Western blot analysis}

Proteins were extracted from intestinal tissues or IEC-6 cells respectively using RIPA lysis buffer (Beyotime, Shanghai, China). Protein concentrations were determined using a BCA Protein Assay kit (Beyotime, Shanghai, China). Proteins were separated using SDS-PAGE and transferred to PVDF membranes. Rabbit polyclonal antibodies were used as primary antibodies against p-JAK2, JAK2, p-STAT3, STAT3 (Abclonal, Woburn, USA), Bcl-2, Bax, cleaved caspase3, occludin (Proteintech, Wuhan, China), and ZO-1 (Santa Cruz, CA, USA). The HRP-conjugated secondary antibody was goat anti-rabbit IgG (Proteintech, Wuhan, China) used at $1: 2000$. The ECL western blot detection reagents were used for visualization of the protein bands. And the bands were analyzed using quantity one software (Bio-Rad, USA).

\subsection{Immunofluorescence staining}

The nuclear translocation of p-STAT3 was examined using immunofluorescence staining. IEC-6 cells were cultured in chamber slides and then treated with catalpol at the concentration of $40 \mu \mathrm{M}$. The cells were washed with PBS and fixed with $4 \%$ paraformaldehyde for $20 \mathrm{~min}$ at room temperature. Samples were permeabilized with $0.4 \%$ TritonX-100 for $10 \mathrm{~min}$, and washed with PBS for three times followed by blocking with $2 \%$ bovine serum albumin (BSA) in PBS at $37^{\circ} \mathrm{C}$ for $1 \mathrm{~h}$. Rabbit anti-p-STAT3 $(1: 50)$ in the $1 \%$ blocking solution was added into the samples and incubated overnight at $4{ }^{\circ} \mathrm{C}$. Following three 5 min washes with PBS, fluorescein-conjugated secondary antibody $(1: 100)$ was added in $1 \%$ blocking solutions and incubated for $1 \mathrm{~h}$. The stained samples were mounted with DAPI $\left(1 \mu \mathrm{g} \mathrm{mL} \mathrm{m}^{-1}\right.$ for $\left.10 \mathrm{~min}\right)$ to stain cell nuclei. After three additional 5 min washes, the samples were examined and analyzed using a fluorescence microscope.

Small intestine sections were incubated with rabbit antioccludin (1:50, Proteintech, Wuhan, China) or anti-ZO-1 ( $1: 50$, Santa Cruz, CA, USA) in a humidified chamber overnight at $4{ }^{\circ} \mathrm{C}$. After washing with PBS for three times, the sections were incubated with a fluorescein-conjugated secondary antibody (1:100, Beyotime Biotechnology, Shanghai) for $1 \mathrm{~h}$. After three additional 5 min washes, the samples were examined and analyzed using a fluorescence microscope.

\subsection{Biochemical analysis of intestinal tissues}

Serum I-FABP and DAO activity were determined using ELISA kits according to the manufacturer's instructions. Small intestine sections of rats were homogenized in ice-cold normal saline. The homogenates were centrifuged at $4000 \mathrm{~g} \mathrm{~min}^{-1}$ at $4{ }^{\circ} \mathrm{C}$ for $10 \mathrm{~min}$. Then pro-inflammatory cytokines and mediators, including IL-6, IL-1 $\beta$, and TNF- $\alpha$, were determined by using ELISA kits. The contents of MDA, SOD, GSH, and MPO in small intestine were measured by using the commercial kits according to the manufacturer's instructions.

\subsection{Small RNA interference transfection}

Transfection of small interfering RNA (siRNA) was used to down-regulate JAK2. IEC-6 cells were plated into 6-well plates for $24 \mathrm{~h}$ and then transfected with JAK2 siRNA or control siRNA (GenePharma, Shanghai, China) using Lipofectamine 2000
A<smiles>OC[C@H]1O[C@H](O[C@@H]2OC=C[C@H]3[C@H](O)[C@H]4O[C@@]34[C@H]2CO)[C@H](O)[C@@H](O)[C@H]1O</smiles>

C

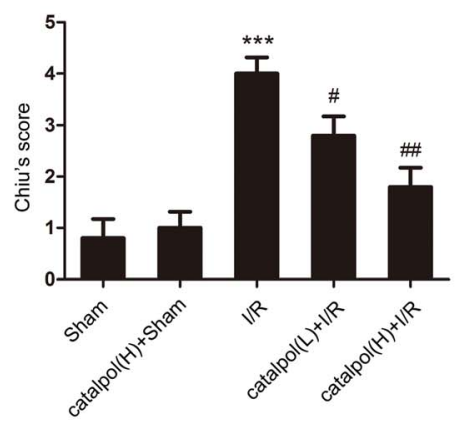

B

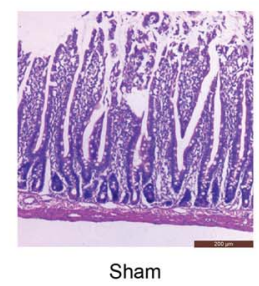

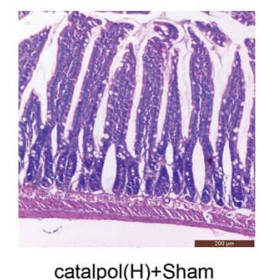

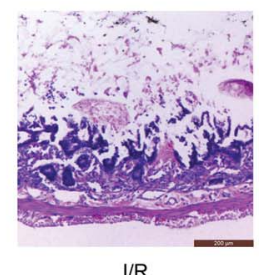

I/R

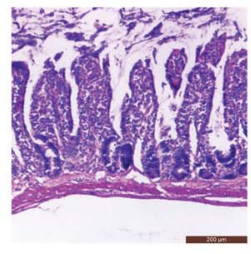

catalpol(L)+l/R

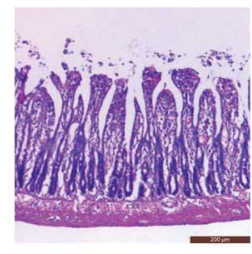

catalpol(H)+l/R

Fig. 1 Catalpol protected against I/R-induced rat intestinal morphologic changes. (A) The chemical structure of catalpol. (B) Representative images of intestinal morphologic changes ( $\mathrm{H} \& \mathrm{E}$ staining, original magnification $\times 200)$. (C) Chiu's scores in different groups. Values are expressed as mean $\pm \mathrm{SEM}(n=8)$. $* * * P<0.001 \mathrm{vs}$. sham group; $\# P<0.05$, \#\#P<0.01 vs. I/R group. 
reagent (Invitrogen, Shanghai, China) according to the manufacturer's instructions. Six hours after transfection, the cells were treated with catalpol before $H / R$ injury, and then the protein levels of JAK2, p-STAT3, STAT3, Bax, Bcl-2, and cleaved caspase 3 were measured.

\subsection{Statistical analysis}

Data are presented as the mean \pm SEM. According to the GraphPad Prism 5.0 software (GraphPad Software Inc., San Diego, CA, USA), a two-tailed Student's $t$-test was used to compare means between two groups. Statistical significance was inferred at $P$-values of less than 0.05 .

\section{Results}

\subsection{Effects of catalpol on rat intestinal $\mathrm{I} / \mathrm{R}$ injury in vivo}

3.1.1. Effects of catalpol on $\mathbf{I} / \mathbf{R}$-induced intestinal morphologic injuries. Morphological signs of small intestine injuries were observed using microscopy. The protective effects of catalpol on histological changes of rat intestinal $\mathrm{I} / \mathrm{R}$ injury were determined by using $\mathrm{H} \& \mathrm{E}$ staining. There were no observable morphologic signs of injuries in the intestinal tissues of both sham rats and catalpol $(\mathrm{H})+$ sham rats. Significant intestinal morphologic alterations were observed in I/Rinjured rats, which manifested as denuded villi, dilated capillaries, digestion and disintegration of lamina propria, hemorrhage, and ulceration. I/R-induced rat intestinal injury was significantly attenuated by catalpol $\left(25,50 \mathrm{mg} \mathrm{kg}{ }^{-1}\right)$ pretreatment (Fig. 1B). Meanwhile, the Chiu's score was significantly increased in I/R-injured rats compared with the sham rats, and catalpol pretreatment significantly reversed the increased the Chiu's score (Fig. 1C), showing the protective effects of catalpol on intestinal $\mathrm{I} / \mathrm{R}$ injury.

3.1.2. Effects of catalpol on intestinal $I / R$-induced oxidative stress. MDA is a biomarker of oxidative damage, ${ }^{25}$ and SOD and GSH are biomarkers of antioxidant capacity. ${ }^{26}$ Compared with sham rats, the intestinal MDA level was significantly increased, and the intestinal SOD and GSH levels were markedly decreased in I/R-injured rats. Catalpol $\left(50 \mathrm{mg} \mathrm{kg}^{-1}\right)$ pretreatment not only significantly reversed the increased MDA level but also significantly reversed the decreased SOD and GSH levels (Fig. 2A). Catalpol did not affect those indicators in catalpol $(\mathrm{H})+$ sham rats.
A

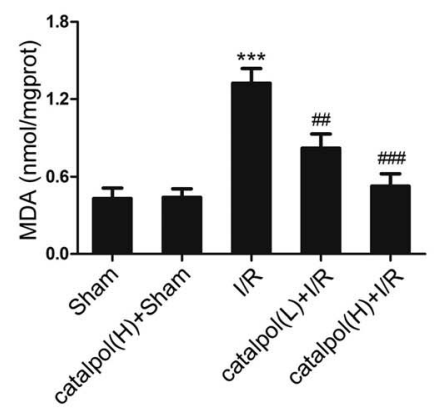

B
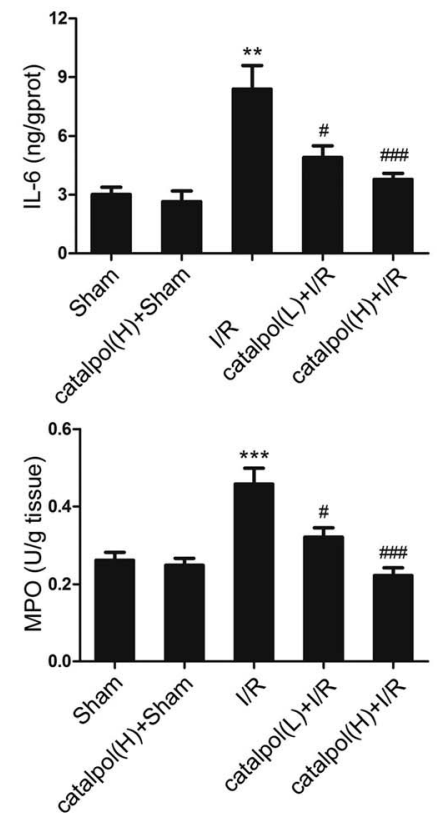
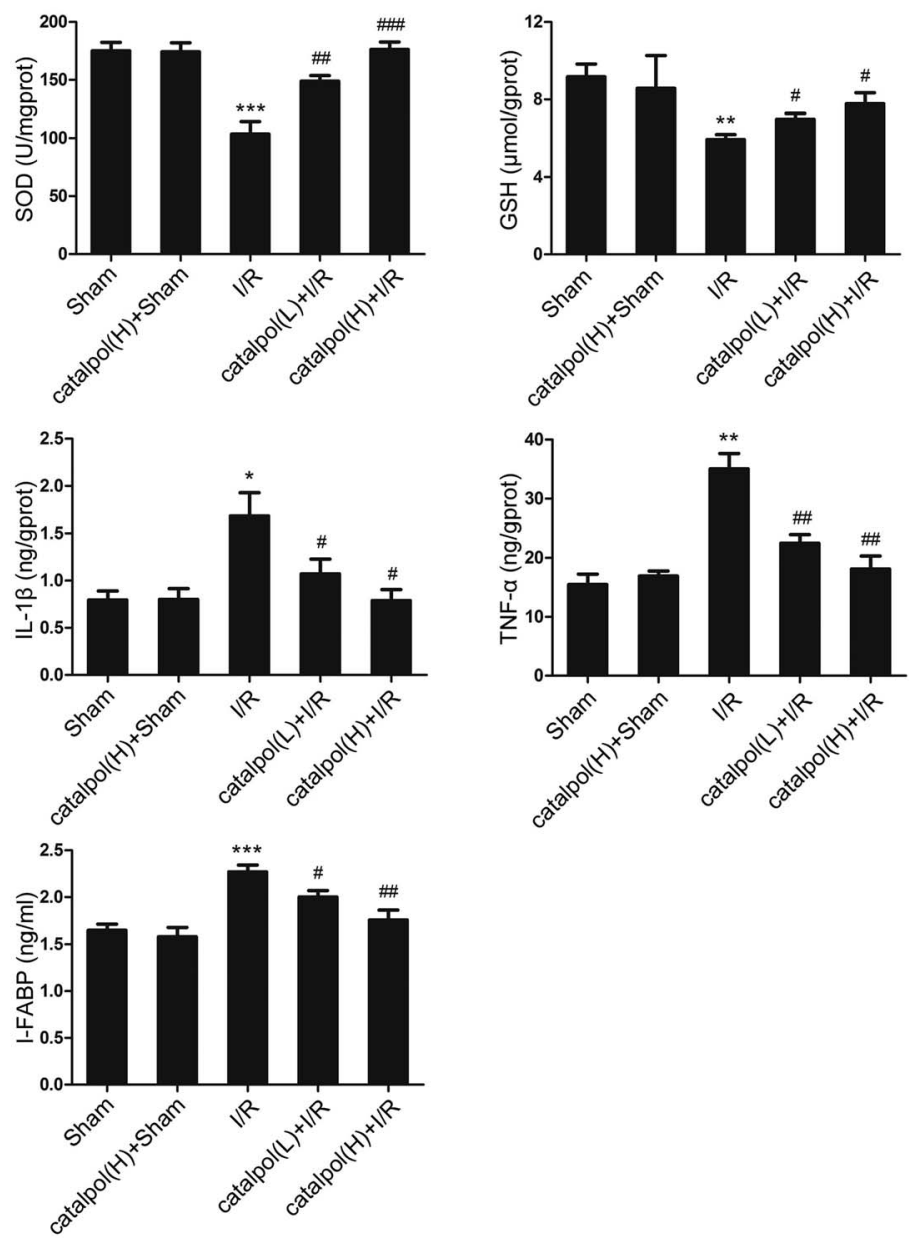

Fig. 2 Catalpol inhibited oxidative stress and inflammation in rat intestinal I/R injury. (A) Effects of catalpol on MDA, SOD, and GSH in intestinal tissue. (B) Effects of catalpol on IL-6, IL-1 $\beta$, TNF- $\alpha$, and MPO in intestinal tissue, and I-FABP in serum. Values are expressed as mean \pm SEM $(n=8)$. $* P<0.05, * * P<0.01, * * * P<0.001$ vs. sham group; $\# P<0.05, \# \# P<0.01$, \#\#\#P<0.001 vs. I/R group. 
3.1.3. Effects of catalpol on intestinal $\mathbf{I} / \mathbf{R}$-induced inflammation. Inflammation induced by intestinal $\mathrm{I} / \mathrm{R}$ is characterized by an increase in MPO activity (an index of neutrophil infiltration), ${ }^{27}$ and increase of inflammatory cytokines including IL-6, IL-1 $\beta$, and TNF- $\alpha .{ }^{28}$ And I-FABP is also an important indicator of both intestinal tissue injury and inflammation. ${ }^{29}$ Our results indicated that I-FABP and other inflammatory parameters including IL-6, IL-1 $\beta$, TNF- $\alpha$, MPO were significantly increased in I/R-injured rats compared with sham rats. Catalpol pretreatment $\left(25,50 \mathrm{mg} \mathrm{\textrm {kg } ^ { - 1 }}\right)$ significantly reversed these increased inflammatory indicators (Fig. 2B). Catalpol did not significantly affect those inflammation-related indicators in catalpol $(\mathrm{H})+$ sham rats.

3.1.4. Effects of catalpol on $I / R$ injured rat intestinal barrier function. DAO is synthesized in epithelial cells of intestinal villi which are usually an epicenter of the ischemia. ${ }^{30}$ And tight junction (TJ) proteins including occludin and ZO-1 are vital for normal intestinal architecture. ${ }^{31}$ The disturbance of the distribution and expression of these proteins are detected in intestinal barrier injuries. In intestinal I/R-injured rats, DAO serum level significantly increased and TJ proteins markedly reduced compared with the sham-operated rats (Fig. 3A). The ELISA result showed that catalpol pretreatment significantly reduced serum DAO level of I/R-injured rats compared with I/Rinjured rats. According to western blot analysis and immunofluorescence assays, the results showed that catalpol pretreatment markedly reversed the decreased expressions of both occludin and ZO-1 proteins in I/R-injured rats compared with intestinal I/R-injured rats (Fig. 3B and C). Catalpol did not significantly affect these indicators in catalpol $(\mathrm{H})+$ sham rats.

3.1.5. Effects of catalpol on intestinal $\mathbf{I} / \mathbf{R}$-induced apoptosis in vivo. Mitochondria-mediated apoptosis plays a central role in tissue homeostasis. ${ }^{32}$ The decrease of Bcl-2/Bax ratio is found to activate the mitochondrial apoptotic pathway, and cleaved caspase 3 is a mark of apoptotic execution protein. ${ }^{33}$ JAK2 activation, following by activation of STAT3, stimulates apoptosis. ${ }^{34}$ Apoptosis induced by intestinal I/R was detected using TUNEL staining and the expression of apoptosis-related
A

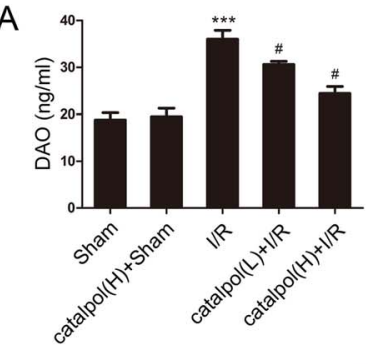

C

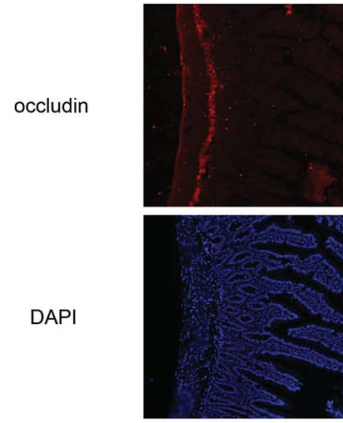

Sham
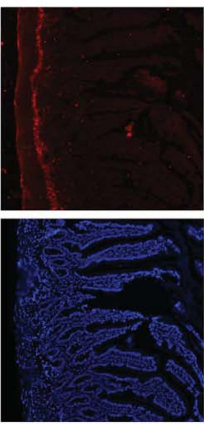

Sham
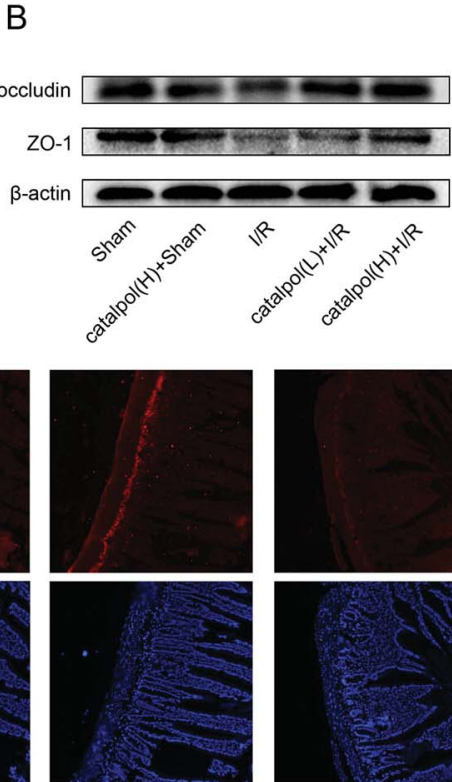

catalpol(H)+l/R
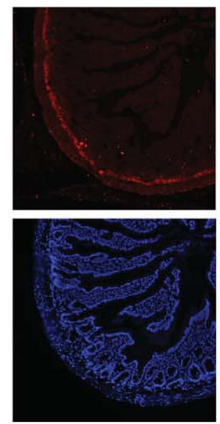

catalpol $(\mathrm{H})+\mathrm{l} / \mathrm{R}$
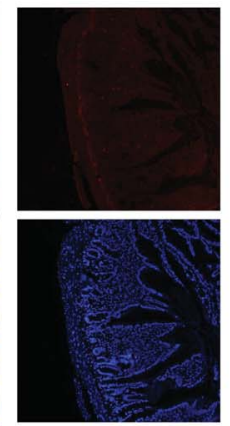

I/R

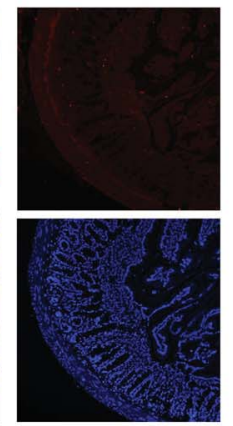

I/R
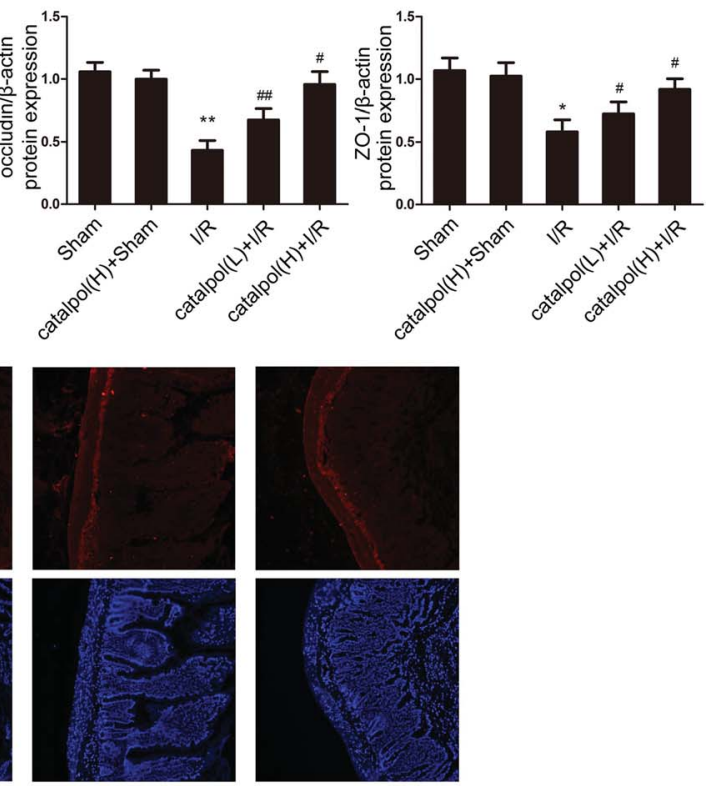

catalpol(L)+l/R
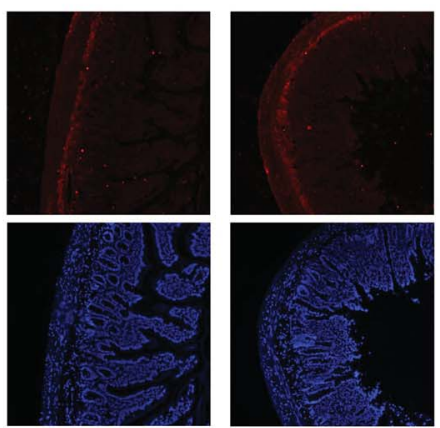

catalpol(H)+l/R

Fig. 3 Catalpol restored intestinal barrier function in I/R-injured rats. (A) The serum DAO level in different groups ( $n=8$ ). (B) The protein expression of occludin and ZO-1 in intestine $(n=3)$. (C) Effects of catalpol on the expression of occludin and ZO-1 using immunofluorescence

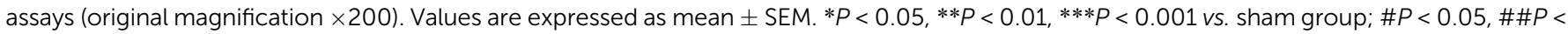
0.01 vs. I/R group. 
proteins was measured using western blot analysis. Our results showed that intestinal I/R significantly increased apoptosis, and catalpol pretreatment significantly reversed the increased apoptosis by reducing the TUNEL-positive cells (Fig. 4A), by increasing Bcl-2/Bax ratio, and by decreasing the expression of cleaved caspase3 (Fig. 4C). Western blot analysis showed that pJAK2 and p-STAT3 were increased in I/R-injured rats and catalpol pretreatment $\left(25,50 \mathrm{mg} \mathrm{kg}^{-1}\right)$ significantly and selectively reversed the increased p-JAK2 and p-STAT3 without affecting total JAK2 and STAT3 (Fig. 4B). Catalpol did not affect those in catalpol $(\mathrm{H})+$ sham rats. The results suggested that JAK2/STAT3 pathway is involved in the protective effects of catalpol on intestinal I/R injury.

\subsection{Effects of catalpol on $\mathbf{H} / \mathbf{R}$-injured IEC-6 cells}

3.2.1. Effects of catalpol pretreatment on cell viability. Cell viability reflects the survival of IEC-6 cells after H/R injury. IEC-6 cells were pretreated with gradient dilution of catalpol in normal and H/R conditions for $12 \mathrm{~h}$. The CCK-8 assay showed that catalpol, at the concentration of $80 \mu \mathrm{M}$ or lower, did not affect the viability of IEC-6 cells (Fig. 5A). Compared with the $\mathrm{H}$ / R-injured control, catalpol pretreatment at concentrations of 10,20 , and $40 \mu \mathrm{M}$ on H/R-injured IEC- 6 cells for $12 \mathrm{~h}$ significantly reversed the decreased cell viability (Fig. 5B and C), indicating that catalpol pretreatment selectively protected against H/R-induced cell injury.

3.2.2. Effects of catalpol on $\mathrm{H} / \mathrm{R}$-induced apoptosis in IEC-6 cells. The modulatory effects of catalpol on apoptosis through inhibiting JAK2/STAT3 pathway observed in vivo were further evaluated by using H/R-injured IEC- 6 cells. Both TUNEL assay and $\mathrm{AO} / \mathrm{EB}$ staining showed that apoptosis was increased in $\mathrm{H}$ / R-injured IEC-6 cells, and catalpol $(10,20,40 \mu \mathrm{M})$ pretreatment reversed the increased apoptosis compared with $\mathrm{H} / \mathrm{R}$-injured IEC-6 cells (Fig. 5D and E). And western blot analysis showed

A
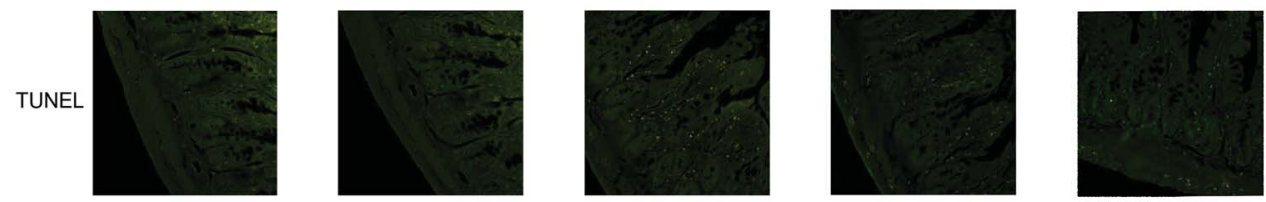

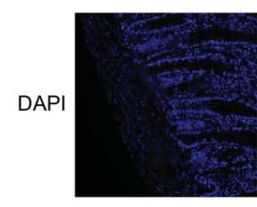

Sham

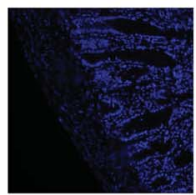

Catalpol(H)+Sham

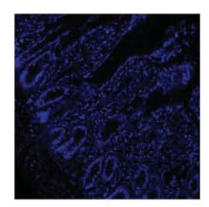

I/R

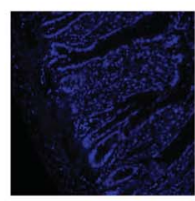

Catalpol $(\mathrm{L})+\mathrm{I} / \mathrm{R}$

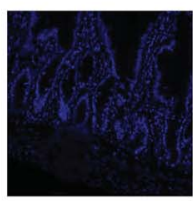

Catalpol(H)+l/R

B
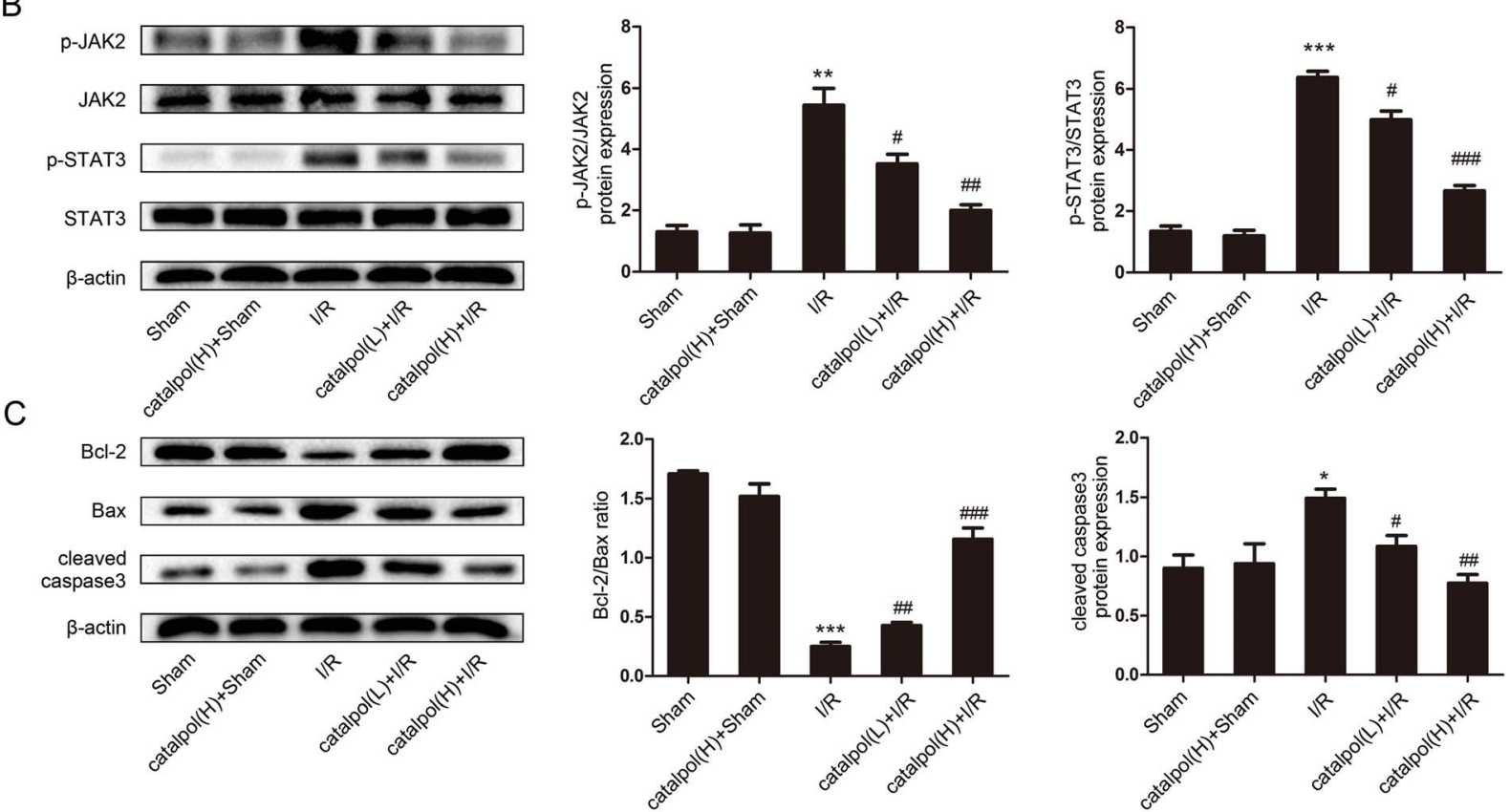

Fig. 4 Catalpol inhibited apoptosis via targeting JAK2/STAT3 signaling pathway in I/R-injured rats. (A) TUNEL staining of intestine after intestinal I/R. (B) Representative western blot analysis of total and phosphorylated JAK2 and total and phosphorylated STAT3 ( $n=3$ ). (C) The protein expression of $\mathrm{Bcl}-2$, Bax and cleaved caspase3 in intestine $(n=3)$. Values are expressed as mean $\pm \mathrm{SEM} . * P<0.05, * * P<0.01, * * * P<0.001$ vs. sham group; $\# P<0.05$, \#\# $<0.01$, \#\# $P<0.001$ vs. I/R group. 
A
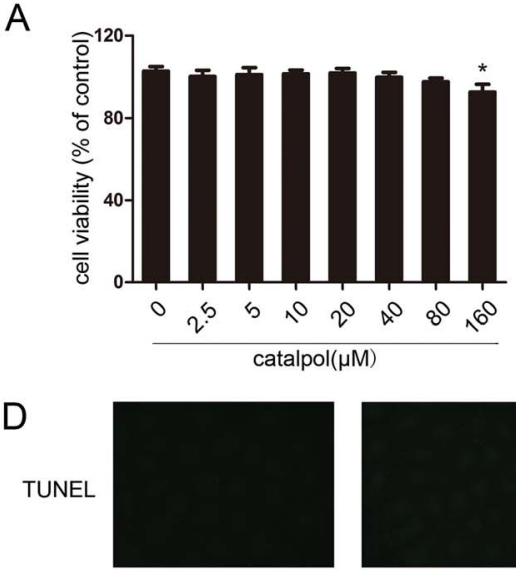

control

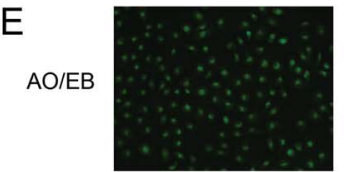

control
B
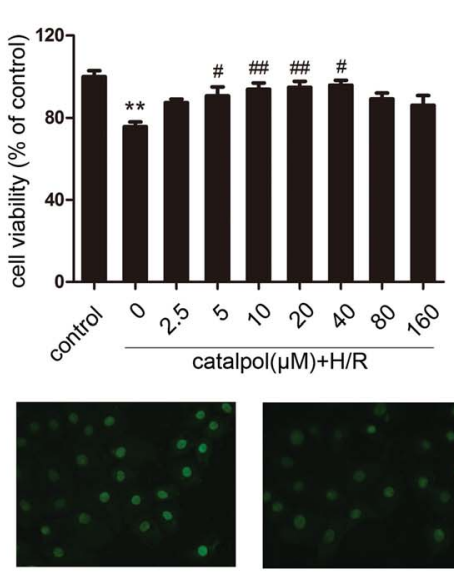

$H / R$

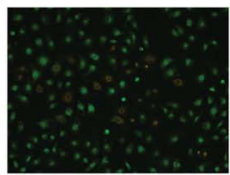

H/R

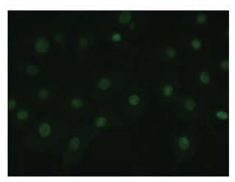

catalpol $(10 \mu \mathrm{M})+\mathrm{H} / \mathrm{R}$

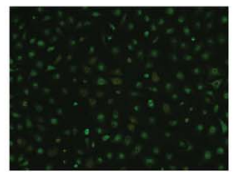

catalpol $(10 \mu \mathrm{M})+\mathrm{H} / \mathrm{R}$
C
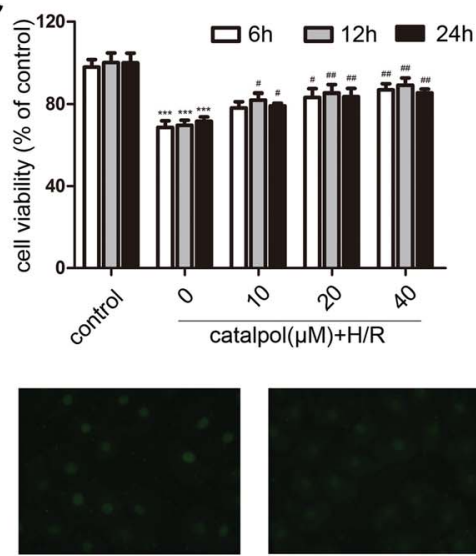

catalpol $(20 \mu \mathrm{M})+\mathrm{H} / \mathrm{R}$

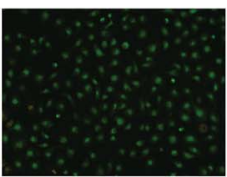

catalpol $(20 \mu M)+H / R$

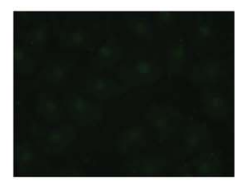

catalpol $(40 \mu \mathrm{M})+\mathrm{H} / \mathrm{R}$

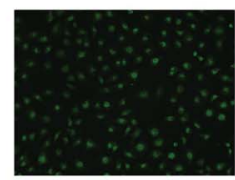

catalpol $(40 \mu \mathrm{M})+\mathrm{H} / \mathrm{R}$

Fig. 5 Catalpol protected IEC-6 cell against H/R injury. (A) Effects of catalpol $(2.5,5,10,20,40,80,160 \mu \mathrm{M})$ on the IEC-6 cell viability for $12 \mathrm{~h}$ in normal condition. (B) Effects of catalpol $(2.5,5,10,20,40,80,160 \mu \mathrm{M})$ on the IEC-6 cell viability for $12 \mathrm{~h}$ in $\mathrm{H} / \mathrm{R}$ condition. (C) Effects of catalpol $(10,20,40 \mu \mathrm{M})$ on the IEC-6 cell viability for $6,12,24 \mathrm{~h}$ in H/R condition. (D) Effects of catalpol on TUNEL assay in IEC-6 cell (original magnification $\times 400$ ). (E) Effects of catalpol on AO/EB staining in IEC-6 cell (original magnification $\times 200$ ). Values are expressed as mean \pm SEM. $* P<0.05, * * P<0.01, * * * P<0.001$ vs. control group; $\# P<0.05, \# \# P<0.01$ vs. H/R group.

that catalpol $(10,20,40 \mu \mathrm{M})$ pretreatment increased Bcl-2/Bax ratio and decreased the expression of pro-apoptotic cleaved caspase 3 compared with control H/R-injured cells (Fig. 6B). Catalpol $(40 \mu \mathrm{M})$ did not affect those in normal IEC-6 cells.

\subsection{Modulation of catalpol on JAK2/STAT3 signaling pathway}

JAK2 and STAT3 phosphorylation in IEC- 6 cells in H/R condition was significantly increased. Catalpol pretreatment significantly and selectively reversed the increased phosphorylation of JAK2 and STAT3 in a dose-dependent manner (Fig. 6A). Immunofluorescence analysis indicated that p-STAT3 nuclear translocation was inhibited by catalpol $(40 \mu \mathrm{M})$ (Fig. 6C).

IEC-6 cells transfected with JAK2 siRNA assayed in $H / R$ condition were used to assess the correlation between catalpol-induced protection and JAK2/STAT3 signaling pathway. In vitro siRNA knockdown of JAK2 significantly decreased JAK2, p-JAK2, STAT3, p-STAT3, and other apoptosis-related proteins including Bcl-2, Bax, and cleaved caspase3, indicating that JAK2 siRNA partially simulated the effects of catalpol. JAK2 siRNA + catalpol did not further decrease JAK2, p-JAK2, STAT3, p-STAT3, and other apoptosis-related proteins, suggesting that inhibition of JAK2/STAT3 signaling pathway plays a key role in catalpolinduced protective effects against intestinal I/R injury.

\section{Discussion}

Intestinal $\mathrm{I} / \mathrm{R}$ injury is characterized with a high morbidity and mortality rate. ${ }^{35,36}$ Its pathophysiological process is typically associated with intestinal and mesenteric vascular dysfunction, which can also occur as a result of surgery, organ transplantation, septic shock, and trauma. ${ }^{37} \mathrm{I} / \mathrm{R}$ may cause intestinal injury and induce systemic circulation of intestinal bacteria and endogenous endotoxins, leading to multiple organ dysfunction syndrome.

Ischemia causes injury and reperfusion may exacerbate the damage. Intestinal $\mathrm{I} / \mathrm{R}$ injury can be caused by inflammatory cytokines, ${ }^{38}$ oxygen free radicals, ${ }^{39}$ and neutrophil infiltration. ${ }^{\mathbf{4 0}}$ And excessive oxidative stress, inflammation damage, and disruption of intestinal epithelial homeostasis, ultimately induce apoptosis. ${ }^{41-44}$ Apoptosis is found to have a vital impact in tissue injury and apoptosis is a major mode of cell death induced by $\mathrm{I} / \mathrm{R}^{\mathbf{4 5 , 4 6}}$ Therefore, anti-inflammation, antioxidation, restoration of barrier function, and inhibition of apoptosis can be selected as the indicators for amelioration of intestinal $\mathrm{I} / \mathrm{R}$ injury.

Our in vivo study showed that catalpol $\left(25,50 \mathrm{mg} \mathrm{kg}{ }^{-1}\right)$ pretreatment effectively alleviated intestinal $\mathrm{I} / \mathrm{R}$ injury by reducing the oxidative stress through reversing the increased MDA level and reversing the decreased SOD and GSH levels in catalpol-treated I/R-injured rats (Fig. 2A). Catalpol pretreatment markedly alleviated the intestinal lesions (Fig. 1B) and significantly ameliorated the inflammation characterized by reversing the increased MPO, IL-6, IL-1 $\beta$, TNF- $\alpha$, and I-FABP (Fig. 2B). And catalpol pretreatment markedly reduced the DAO level and increased the expressions of both occludin and ZO-1 proteins (Fig. 3), showing that catalpol restored intestinal barrier function in intestinal I/R-injured rats. All of these in vivo results 

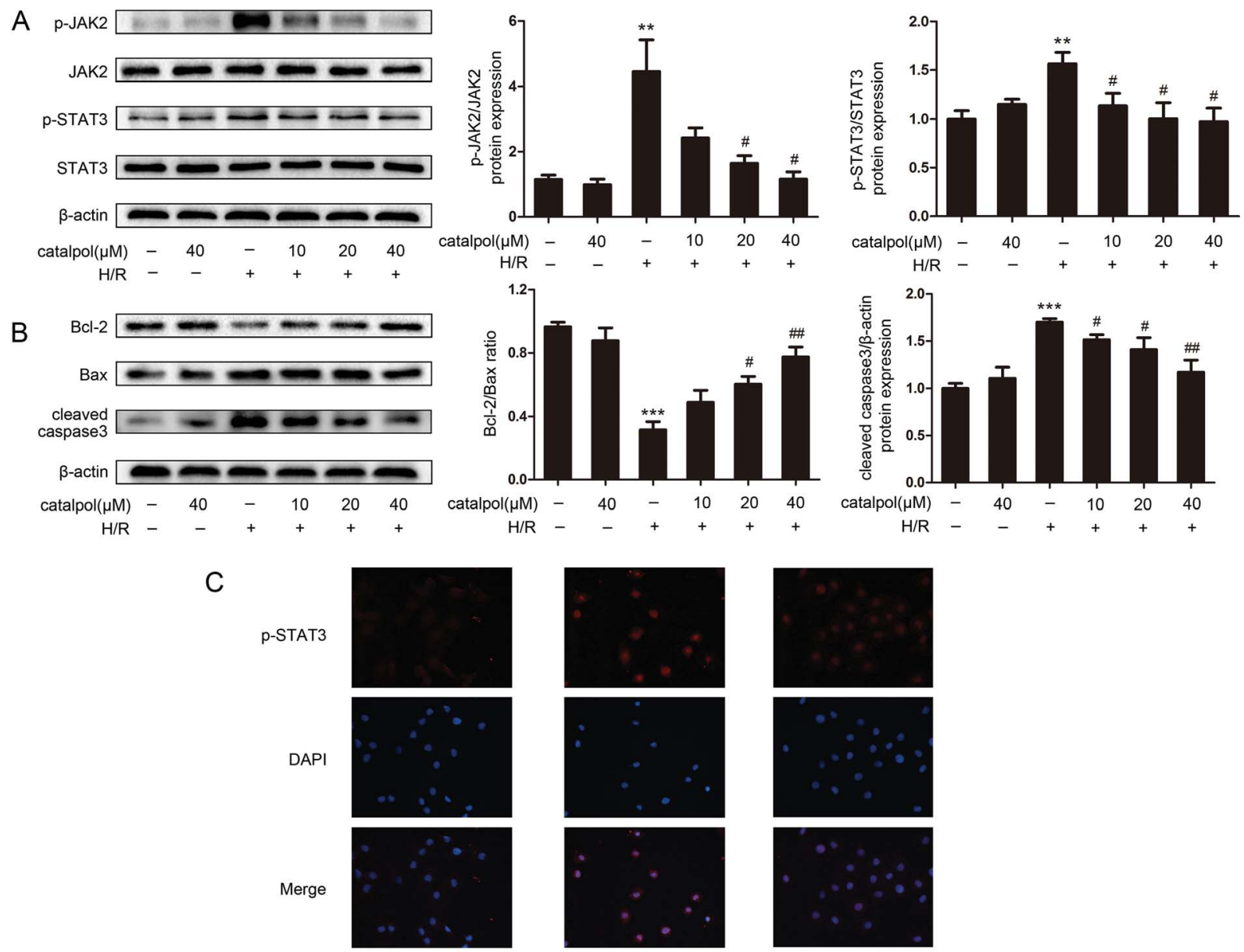

catalpol $(\mu \mathrm{M})$

-

$-$

40

Fig. 6 Catalpol inhibited JAK2/STAT3 signaling pathway in H/R-injured IEC- 6 cells. (A) Effects of catalpol on the expression of p-JAK2, JAK2, pSTAT3, STAT3 in IEC- 6 cells in normal and H/R conditions $(n=3)$. (B) Effects of catalpol on the expression of Bcl-2, Bax and cleaved caspase3 in IEC- 6 cells in normal and H/R conditions $(n=3)$. (C) Effects of catalpol on p-STAT3 translocation in IEC- 6 cells in normal and H/R conditions

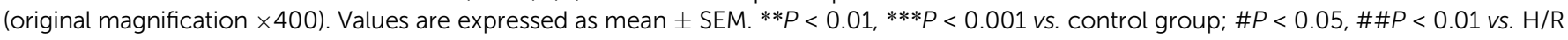
group.

indicated that catalpol inhibited both inflammation and oxidative stress and restored intestinal barrier function.

Mitochondria play an important role in energy production and reactive oxygen homeostasis, ${ }^{47}$ triggering apoptotic events. ${ }^{48}$ Our in vivo results indicated that catalpol pretreatment reduced TUNEL-positive cells, increased Bcl-2/Bax ratio and decreased the expression of cleaved caspase 3 (Fig. 4A and C), showing that catalpol markedly alleviated intestinal $\mathrm{I} / \mathrm{R}$ injury by inhibiting apoptosis. The in vivo inhibitory effects of catalpol on apoptosis were also observed in vitro. TUNEL assay (Fig. 5C), AO/EB staining (Fig. 5D), and western blot analysis (Fig. 6A and B) indicated that catalpol significantly prevented the occurrence of apoptosis.

JAK2/STAT3 signaling pathway plays a major role in cytokine signal transduction of various cytokines and growth factors as IL-6 (ref. 49 and 50) and this signal pathway is activated during the tissue injuries and closely related to oxidative stress, inflammation, and apoptosis. ${ }^{12,51-53}$ Activation of JAK2/STAT3 signaling pathway was observed in intestinal $\mathrm{I} / \mathrm{R}$ injury, ${ }^{\mathbf{1 2}}$ however the detailed mechanism of JAK2/STAT3 in I/R-induced apoptosis needs to be further explored. Our results from both in vivo and in vitro studies showed that catalpol pretreatment (25, $50 \mathrm{mg} \mathrm{kg}^{-1}$ ) significantly and selectively reversed the increased p-JAK2 and p-STAT3 without affecting total JAK2 and STAT3 (Fig. 4B and 6A), indicating that catalpol-induced anti-apoptotic effects on intestinal $\mathrm{I} / \mathrm{R}$ was mediated through selective inhibiting p-JAK2 and p-STAT3. Furthermore, in vitro study showed that catalpol inhibited p-STAT3 nuclear translocation, resulting in a decrease of apoptosis (Fig. 6C).

To verify the relationship between catalpol-induced protection and JAK2/STAT3 signaling pathway, JAK2 siRNA was transfected into IEC- 6 cells under H/R condition. JAK2 siRNA decreased the expression of JAK2, p-JAK2, STAT3, p-STAT3, and inhibited mitochondria-mediated apoptosis (Fig. 7). And JAK2 siRNA + catalpol could not further decrease the expression of JAK2, p-JAK2, and other apoptosis-related proteins (Fig. 7), suggesting that JAK2/STAT3 signaling pathway plays a key role in catalpol-induced anti-apoptotic effect. 

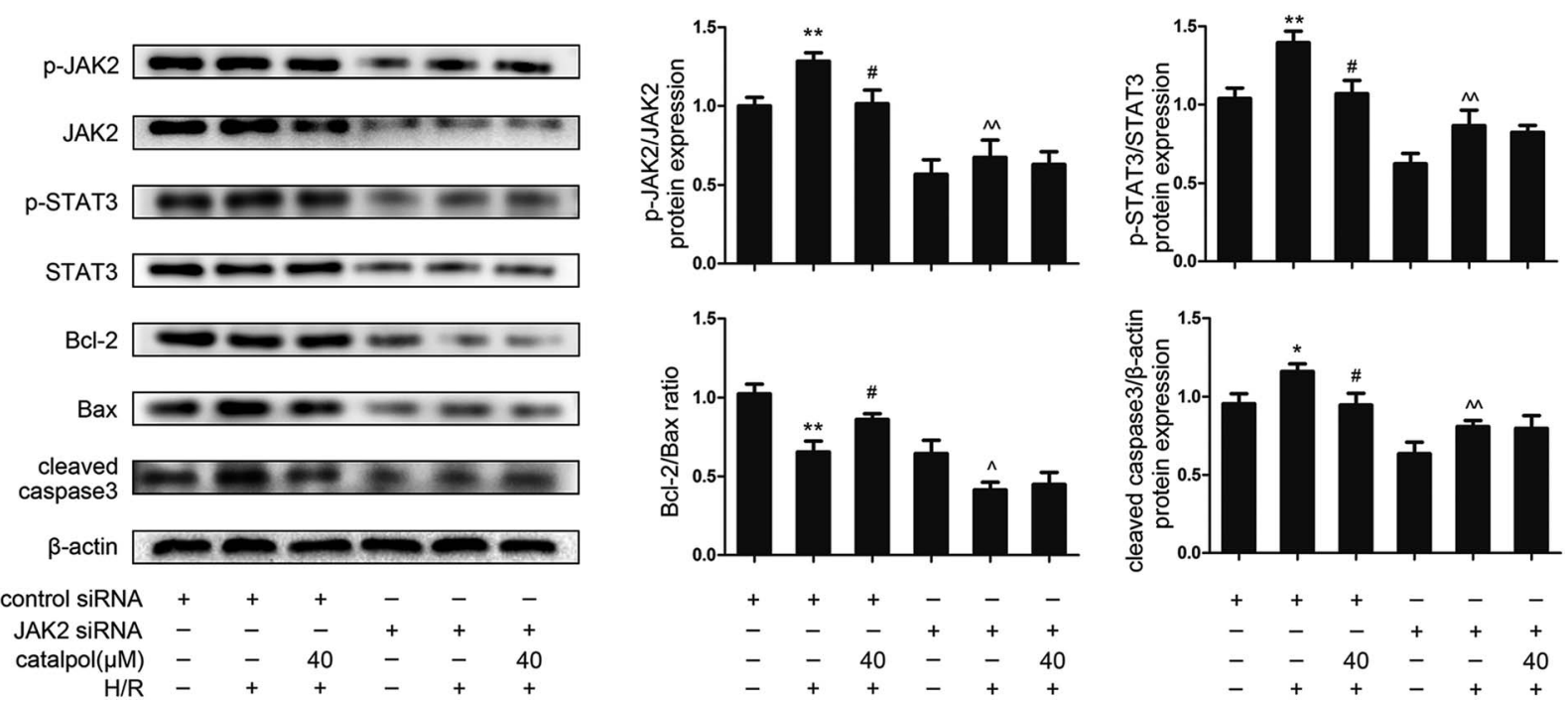

Fig. 7 siRNA knockdown of JAK2 in H/R-injured IEC- 6 cell blocked the catalpol-induced reduction in $\mathrm{p}-\mathrm{JAK} 2$ level and apoptotic rate under $\mathrm{H} / \mathrm{R}$ condition. Values are expressed as mean $\pm \operatorname{SEM}(n=3)$. $* P<0.05, * * P<0.01$ vs. control group; $\# P<0.05$ vs. $\mathrm{H} / \mathrm{R}$ group; $\wedge P<0.05, \wedge \wedge P<0.01$ vs. proteins expression before $\mathrm{H} / \mathrm{R}$ in siRNA knockdown of JAK2.

In summary, the present study demonstrated that catalpol pretreatment significantly attenuated rat intestinal I/R injury by inhibiting oxidative stress and inflammation, restoring intestinal barrier function, and decreasing mitochondria-mediated apoptosis through blocking JAK2/STAT3 signaling pathway via selective inhibition of p-JAK2. Catalpol did not significantly affect the corresponding controls. Furthermore, catalpol is low toxicity, and has anti-bacterial activity which may inhibit bacterial infection during the development of intestinal $\mathrm{I} / \mathrm{R}$ injury. Our study suggests that p-JAK2 plays a key role in catalpol-induced protection on intestinal $\mathrm{I} / \mathrm{R}$ injury and $\mathrm{p}$-JAK2 can be chosen as a therapeutic target, providing valuable information for the further translational studies of catalpol in protecting against intestinal I/R injury.

\section{Conflicts of interest}

The authors have declared no conflict of interest.

\section{Acknowledgements}

This study was supported by the National Natural Science Foundation of China (Grant No. 30772601).

\section{References}

1 S. Acosta and M. Bjorck, Br. J. Surg., 2014, 101, e100-108.

2 Q. Zhu, G. He, J. Wang, Y. Wang, W. Chen and T. Guo, Oncotarget, 2017, 8, 13678-13689.

3 Q. Zhu, G. He, J. Wang, Y. Wang and W. Chen, Clinical Science, 2017, 131, 1123-1136.

4 M. R. Pope, U. Bukovnik, J. M. Tomich and S. D. Fleming, J. Immunol., 2012, 189, 5047-5056.

5 M. Kim, S. W. Park, M. Kim, V. D. D'Agati and H. T. Lee, Ann. Surg., 2012, 255, 492-503.
6 Y. Jiang, Z. Zhou, Q. T. Meng, Q. Sun, W. Su, S. Lei, Z. Xia and Z. Y. Xia, Oxid. Med. Cell. Longevity, 2015, 2015, 843721.

7 C. Liu, C. Zhu, G. Wang, R. Xu and Y. Zhu, Inflammation Res., 2015, 64, 395-403.

8 L. W. Hansen, A. Khader, W. L. Yang, J. M. Prince, J. M. Nicastro, G. F. Coppa and P. Wang, Shock, 2016, 45, 359-366.

9 G. Wang, J. Yao, Z. Li, G. Zu, D. Feng, W. Shan, Y. Li, Y. Hu, Y. Zhao and X. Tian, Antioxid. Redox Signaling, 2016, 24, 961-973.

$10 \mathrm{~J}$. Zhu, X. Chen, H. Wang and Q. Yan, Int. J. Clin. Exp. Med., 2015, 8, 2038-2044.

11 F. Roviezzo, S. Cuzzocrea, A. Di Lorenzo, V. Brancaleone, E. Mazzon, R. Di Paola, M. Bucci and G. Cirino, Br. J. Pharmacol., 2007, 151, 377-383.

12 S. H. Wen, Y. Li, C. Li, Z. Q. Xia, W. F. Liu, X. Y. Zhang, W. L. Lei, W. Q. Huang and K. X. Liu, Shock, 2012, 38, 411-419.

13 L. L. Xiong, Y. Tan, H. Y. Ma, P. Dai, Y. X. Qin, R. A. Yang, Y. Y. Xu, Z. Deng, W. Zhao, Q. J. Xia, T. H. Wang and Y. H. Zhang, Int. Immunopharmacol., 2016, 38, 54-60.

14 D. Y. Zheng, M. Zhou, J. Jin, M. He, Y. Wang, J. Du, X. Y. Xiao, P. Y. Li, A. Z. Ye, J. Liu and T. H. Wang, Mediators Inflammation, 2016, 2016, 9348037.

15 R. Zhang, Y. Zhao, Y. Sun, X. Lu and X. Yang, J. Agric. Food Chem., 2013, 61, 7786-7793.

16 J. H. Tao, M. Zhao, D. G. Wang, C. Yang, L. Y. Du, W. Q. Qiu and S. Jiang, J. Chromatogr. B: Anal. Technol. Biomed. Life Sci., 2016, 1009-1010, 163-169.

17 W. Dong, Y. Xian, W. Yuan, Z. Huifeng, W. Tao, L. Zhiqiang, F. Shan, F. Ya, W. Hongli, W. Jinghuan, Q. Lei, Z. Li and Q. Hongyi, J. Ethnopharmacol., 2016, 191, 169-179.

18 P. Zhu, Y. Wu, A. Yang, X. Fu, M. Mao and Z. Liu, Biomed. Pharmacother., 2017, 95, 68-76.

19 W. Chen, X. Li, L. Q. Jia, J. Wang, L. Zhang, D. Hou, J. Wang and L. Ren, Br. J. Pharmacol., 2013, 169, 1140-1152. 
20 X. W. Zheng, W. T. Yang, S. Chen, Q. Q. Xu, C. S. Shan, G. Q. Zheng and J. C. Ruan, Oxid. Med. Cell. Longevity, 2017, 2017, 5058609.

21 M. Zhao, J. Tao, D. Qian, P. Liu, E. X. Shang, S. Jiang, J. Guo, S. L. Su, J. A. Duan and L. Du, J. Chromatogr. B: Anal. Technol. Biomed. Life Sci., 2016, 1009-1010, 122-129.

22 H. F. Zhu, D. Wan, Y. Luo, J. L. Zhou, L. Chen and X. Y. Xu, Int. J. Biol. Sci., 2010, 6, 443-453.

23 R. L. Vannette and T. Fukami, Ecology, 2016, 97, 1410-1419.

24 D. G. Souza, V. Pinho, A. C. Soares, T. Shimizu, S. Ishii and M. M. Teixeira, Br. J. Pharmacol., 2003, 139, 733-740.

25 Y. Hu, X. Tao, X. Han, L. Xu, L. Yin, Y. Qi, Y. Zhao, Y. Xu, C. Wang and J. Peng, Chem.-Biol. Interact., 2016, 258, 234244.

26 L. Monzo-Beltran, A. Vazquez-Tarragon, C. Cerda, P. GarciaPerez, A. Iradi, C. Sanchez, B. Climent, C. Tormos, A. Vazquez-Prado, J. Girbes, N. Estan, S. Blesa, R. Cortes, F. J. Chaves and G. T. Saez, Redox Biol., 2017, 12, 389-402.

27 A. Ahmad and C. Szabo, Pharmacol. Res., 2016, 113, 348-355. 28 D. G. Souza, D. Esser, R. Bradford, A. T. Vieira and M. M. Teixeira, Br. J. Pharmacol., 2005, 145, 1027-1034.

29 D. L. Sun, Y. Y. Cen, S. M. Li, W. M. Li, Q. P. Lu and P. Y. Xu, Sci. Rep., 2016, 6, 34371.

30 X. Chi, W. Yao, H. Xia, Y. Jin, X. Li, J. Cai and Z. Hei, Oxid. Med. Cell. Longevity, 2015, 2015, 986075.

31 L. Gonzalez-Mariscal, R. Tapia and D. Chamorro, Biochim. Biophys. Acta, Biomembr., 2008, 1778, 729-756.

32 Y. Shi, Nat. Struct. Biol., 2001, 8, 394-401.

33 S. Liu, Z. Sun, P. Chu, H. Li, A. Ahsan, Z. Zhou, Z. Zhang, B. Sun, J. Wu, Y. Xi, G. Han, Y. Lin, J. Peng and Z. Tang, Apoptosis, 2017, 22, 672-680.

34 X. J. Li, Q. Y. Ma, Y. M. Jiang, X. H. Bai, Z. Y. Yan, Q. Liu, Q. X. Pan, Y. Y. Liu and J. X. Chen, Sci. Rep., 2017, 7, 353.

35 Z. Liu, J. Jiang, Q. Yang, Y. Xiong, D. Zou, C. Yang, J. Xu and H. Zhan, Cell Death Dis., 2016, 7, e2210.

36 C. L. Chang, P. H. Sung, C. K. Sun, C. H. Chen, H. J. Chiang, T. H. Huang, Y. L. Chen, Y. Y. Zhen, H. T. Chai, S. Y. Chung, M. S. Tong, H. W. Chang, H. H. Chen and H. K. Yip, J. Pineal Res., 2015, 59, 206-220.

37 T. Gobbetti, S. Ducheix, P. le Faouder, T. Perez, F. Riols, J. Boue, J. Bertrand-Michel, M. Dubourdeau, H. Guillou, M. Perretti, N. Vergnolle and N. Cenac, Br. J. Pharmacol., 2015, 172, 910-923.
38 H. Yan, Y. Jihong, Z. Feng, X. Xiaomei, Z. Xiaohan, W. Guangzhi, M. Zhenhai, G. Dongyan, M. Xiaochi, F. Qing, L. Kexin and T. Xiaofeng, Crit. Care Med., 2014, 42, e373-381.

39 O. L. Woodman, R. Long, S. Pons, N. Eychenne, A. Berdeaux and D. Morin, Pharmacol. Res., 2014, 81, 26-33.

40 S. Bertoni, V. Arcaro, V. Vivo, A. Rapalli, M. Tognolini, A. M. Cantoni, F. Saccani, L. Flammini, G. Domenichini, V. Ballabeni and E. Barocelli, Pharmacol. Res., 2014, 81, 1725.

41 A. Bhattacharyya, R. Chattopadhyay, S. Mitra and S. E. Crowe, Physiol. Rev., 2014, 94, 329-354.

42 M. Xian, H. Cao, J. Cao, X. Shao, D. Zhu, N. Zhang, P. Huang, W. Li, B. Yang, M. Ying and Q. He, Int. J. Cancer, 2017, 141, 1029-1041.

43 S. L. Jian, W. W. Chen, Y. C. Su, Y. W. Su, T. H. Chuang, S. C. Hsu and L. R. Huang, Cell Death Dis., 2017, 8, e2779.

44 K. Rashid, S. Chowdhury, S. Ghosh and P. C. Sil, Biochem. Pharmacol., 2017, 143, 140-155.

45 G. Zu, J. Guo, N. Che, T. Zhou and X. Zhang, Sci. Rep., 2016, 6, 38480 .

46 J. Zhou, W. Q. Huang, C. Li, G. Y. Wu, Y. S. Li, S. H. Wen, W. L. Lei and K. X. Liu, Crit. Care Med., 2012, 40, 2438-2448.

47 K. Boengler, M. Kosiol, M. Mayr, R. Schulz and S. Rohrbach, J. Cachexia Sarcopenia Muscle, 2017, 8, 349-369.

48 K. Ishikawa, K. Takenaga, M. Akimoto, N. Koshikawa, A. Yamaguchi, H. Imanishi, K. Nakada, Y. Honma and J. Hayashi, Science, 2008, 320, 661-664.

49 N. Yang, M. Luo, R. Li, Y. Huang, R. Zhang, Q. Wu, F. Wang, Y. Li and X. Yu, Nephrol., Dial., Transplant., 2008, 23, 91-100.

50 E. Mascareno, M. El-Shafei, N. Maulik, M. Sato, Y. Guo, D. K. Das and M. A. Siddiqui, Circulation, 2001, 104, 325-329.

51 D. Guo, J. R. Li, Y. Wang, L. S. Lei, C. L. Yu and N. N. Chen, Acta Pharmacol. Sin., 2014, 35, 770-778.

52 J. E. Jung, H. Karatas, Y. Liu, A. Yalcin, J. Montaner, E. H. Lo and K. van Leyen, J. Cereb. Blood Flow Metab., 2015, 35, 20432051.

53 T. Higuchi, T. Shiraishi, T. Shirakusa, S. Hirayama, H. Shibaguchi, M. Kuroki, M. Hiratuka, S. Yamamoto, A. Iwasaki and M. Kuroki, J. Heart Lung Transplant., 2005, 24, 1557-1564. 